\title{
Evaluación de una Instalación de Refrigeración por Absorción con Energía Solar
}

\author{
Carlos Monné $^{(1)}$, Sergio Alonso ${ }^{(1)}$, Fernando Palacín ${ }^{(2)}$ \\ (1) Área de Máquinas y Motores Térmicos, Departamento de Ingeniería Mecánica, \\ Universidad de Zaragoza. C/ María de luna s/n 50018, Zaragoza-España. \\ (e-mail: cmmb@unizar.es, sealonso@unizar.es) \\ (2) Departamento de Arquitectura Bioclimática, Centro Nacional de Energías Renovables \\ (CENER). Ciudad de la Innovación, nº $7 \cdot 31621$ Sarriguren, Navarra-España. \\ (e-mail: fpalacin@cener.com )
}

Recibido Jul. 28, 2010; Aceptado Sep. 30, 2010; Versión Final recibida Oct. 22, 2010

\section{Resumen}

Se ha evaluado el comportamiento de una instalación de refrigeración solar por absorción, acoplada con un sistema de disipación geotérmica. La metodología de estudio se ha basado en el análisis de los valores registrados con sondas de temperatura, caudal y radiación, durante el período 2007-2009. Los registros indican que el coeficiente de funcionamiento varió entre 0.47 y 0.56 y la potencia de refrigeración producida entre 3.5 y $5.8 \mathrm{~kW}$. Estos resultados se han comparado con los de la misma instalación pero que disipa calor por medio de una torre de refrigeración seca (aerotermo). Se ha comprobado que la instalación de refrigeración se comporta de un modo más estable utilizando el sistema de disipación geotérmico. Sin embargo, para bajas temperaturas, el uso del aerotermo es más eficiente.

Palabras clave: refrigeración solar, ciclo de absorción, disipación geotérmica, ahorro energético.

\section{Evaluation of an Absorption Refrigeration System with Solar Energy}

\begin{abstract}
The performance of a solar cooling absorption installation, coupled with a geothermal heat rejection system has been evaluated. The methodology was based on the analysis of the values recorded by temperature, flow and radiation probes, during the period 2007-2009. The data obtained show that the coefficient of performance varied between 0.47 and 0.56 and the cooling capacity produced varied between 3.5 and $5.8 \mathrm{~kW}$. These results have been compared with those of the same installation but in which heat was dissipated using a dry cooling tower. The study demonstrated that the cooling system behaves in a more stable manner using the geothermal heat rejection system. However, for low temperatures, the use of the dry cooling tower is more efficient.
\end{abstract}

Keywords: solar cooling, absorption cycle, geothermal heat rejection, energy saving. 


\section{INTRODUCCIÓN}

Hoy en día, en España, la mayoría de los edificios han sido diseñados con instalaciones de calefacción, pero no cuentan con equipos de refrigeración. Las necesidades de refrigeración se cubren con las máquinas de aire acondicionado que se alimentan eléctricamente. Debido a la operación de estas máquinas, en verano, el consumo eléctrico alcanza valores elevados, incluso llegando a causar problemas puntuales en las red eléctrica. Por lo tanto, es necesario buscar equipos de refrigeración alternativos que se puedan operar a partir de energías renovables 0 residuales. Una de estas alternativas es el sistema de refrigeración por absorción con apoyo solar, que utiliza energía solar térmica para refrigerar, utilizando un ciclo de absorción. El uso de la energía solar para refrigerar es especialmente interesante porque las horas de más radiación solar son las horas con la demanda de refrigeración más alta (Herold et al, 1996).

Si se comparan los sistemas de refrigeración por absorción con los de compresión, los primeros no son competitivos económicamente a día de hoy, pero son respetuosos con el medio ambiente, y tienen pocas emisiones de $\mathrm{CO}_{2}$. Sin embargo, las máquinas de absorción de baja potencia no han sido ampliamente comercializadas, ya que son caras, y la mayoría de ellas necesitan torres de refrigeración para funcionar. Por lo tanto, la investigación y el desarrollo son necesarios con el fin de reducir el costo de usar el aire acondicionado a partir de la energía solar en los edificios (Balaras et al, 2007). Hay una gran cantidad de investigaciones que se refiere a los estudios teóricos (García, 2006; Assilzadeh et al., 2005; Henning, 2006; Kaynakli y Kilic, 2007) y se han llevado a cabo numerosas simulaciones con TRNSYS (Argiriou et al., 2005; Balghouthi et al., 2005; Atmaca y Yigit, 2003) para este tipo de sistemas, pero la información de los estudios experimentales en este tipo de instalaciones es escasa (Syed et al, 2005).

Este trabajo describe una instalación de refrigeración solar basada en el funcionamiento de una máquina de absorción. La instalación está ubicada en Zaragoza (España) y sirve para refrigerar un pequeño gimnasio del Servicio de Actividades Deportivas (SAD) en la Universidad de Zaragoza. Antes de implementar esta instalación, el campo de colectores solares sólo se utilizaba para el suministro de agua caliente sanitaria y existían problemas de recalentamiento en los colectores debido al sobredimensionamiento de estos. Con la instalación de refrigeración solar, este problema se resolvió y, desde 2007 hasta 2009 se ha venido analizando el comportamiento del sistema. El presente trabajo resume los resultados obtenidos mediante el análisis de los datos registrados en la instalación. Estos siguen la misma línea que los obtenidos en un estudio preliminar de esta instalación (Monné et al., 2008). La investigación demuestra la gran influencia de la temperatura ambiente en el funcionamiento de la instalación.

\section{DESCRIPCIÓN DE LA INSTALACIÓN}

El elemento central de esta instalación es la máquina de absorción Solar045 de Rotártica. Existen tres flujos de agua externos que intercambian calor con distintas partes de la máquina de absorción: el flujo de agua caliente (procedente del intercambiador) que cede calor en el generador, el flujo de agua fría que produce la refrigeración (absorbe calor de la sala y lo cede en el evaporador de la máquina de absorción), y por último, el flujo de agua que absorbe el calor residual del condensador y del absorbedor, cediéndolo al ambiente a través del aerotermo de la instalación. Dicho aerotermo evita la presencia de una torre de refrigeración, suprimiendo así la posible aparición de Legionella. El campo de colectores solares planos utilizado está formado por 15 unidades de $2,5 \mathrm{~m}^{2}$ de superficie útil. Los colectores, junto con una bomba de impulsión, y las tuberías que los comunican con el intercambiador instalado, forman el circuito primario de la instalación por el que circula la mezcla correspondiente de agua y anticongelante. El circuito secundario comunica el intercambiador de calor con la máquina de absorción, en concreto con el generador de dicha máquina. La instalación cuenta además con una unidad Siemens PXC52 y un controlador Web Siemens PXG80-WN con los que se ha realizado la captación de datos de la instalación. Este sistema de control y supervisión es el encargado de recopilar los datos generados por las distintas sondas de medición ubicadas en distintos puntos de la instalación. Así, se tienen mediciones de temperaturas, caudales, radiación solar, etc. 


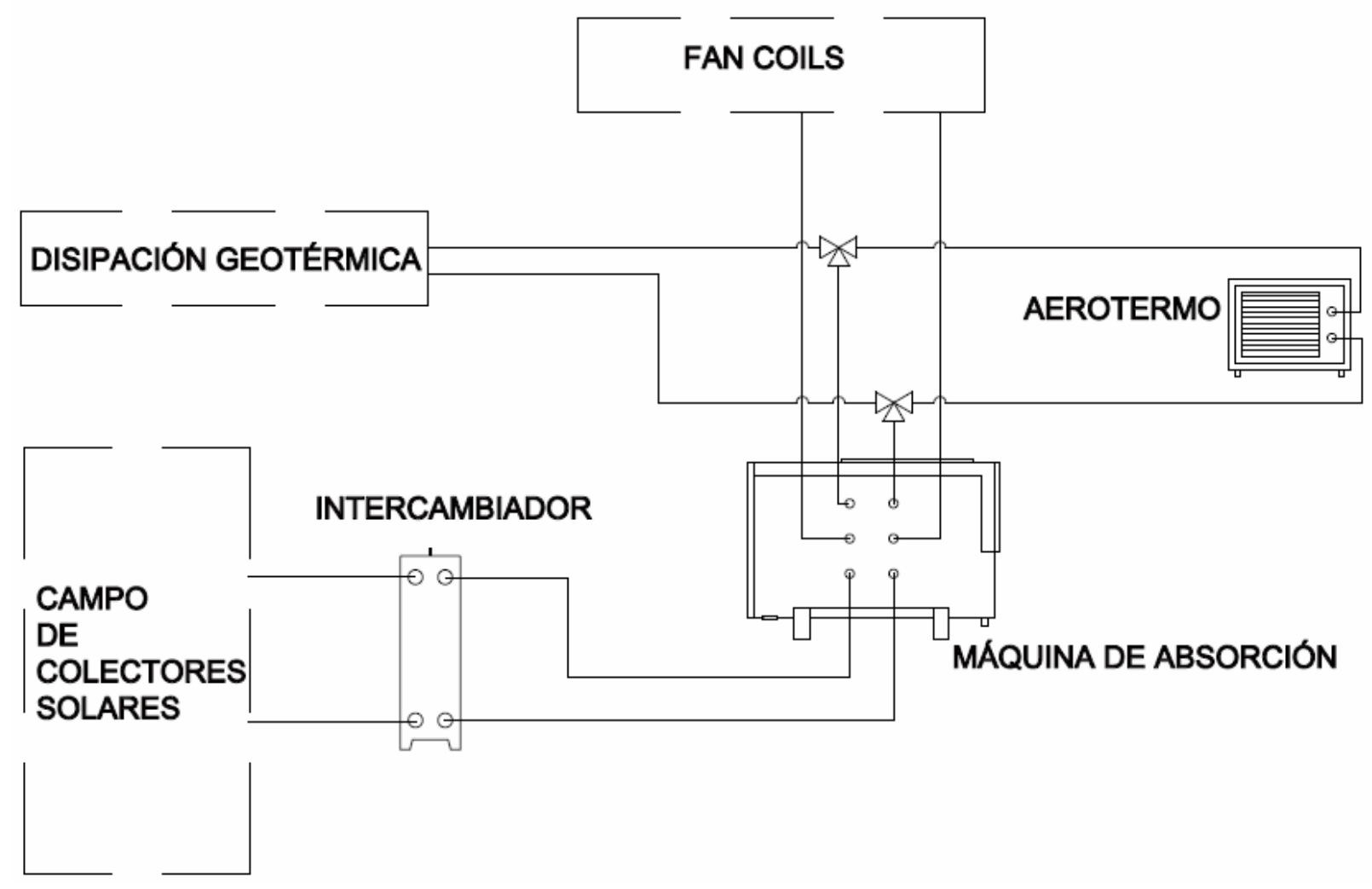

Fig. 1: Esquema de la instalación

Nuevo sistema de disipación de calor

En 2009 se modificó la instalación con un sistema de disipación geotérmica. Se decidió utilizar un foco frío con una temperatura más constante (intercambio geotérmico) para la evacuación del calor producido en el ciclo de absorción. Se realizaron estudios de viabilidad técnica y económica para distintas tipologías de sistemas geotérmicos y finalmente se adoptó como sistema disipativo el sistema geotérmico con circuito abierto.

El nuevo circuito aprovecha un aljibe de agua de unos $25 \mathrm{~m}^{3}$ sito en las cercanías de la instalación de refrigeración solar y que es utilizado como aljibe de riego. El aljibe es alimentado por un pozo subterráneo. El agua del aljibe se renueva diariamente en verano, puesto que se utiliza para regar las zonas de césped situadas en los aledaños del edificio del SAD. Por tanto, la temperatura del agua se mantendrá prácticamente constante, eliminándose de este modo posibles problemas de saturación térmica. El nuevo sistema de disipación extrae el calor del ciclo de absorción por medio de un intercambiador de $20 \mathrm{~kW}$. La distancia total del nuevo trazado es de 190.5 metros, de los cuales unos 90.5 metros del ramal de retorno hacia el aljibe se ha divido en tres tuberías de menor diámetro con el fin de que actúe como un intercambiador geotérmico horizontal. Por lo tanto la disipación del calor generado por el ciclo de absorción se efectuará en dos focos fríos: el aljibe de riego y el intercambiador geotérmico.

Tras la modificación del nuevo sistema disipativo, la instalación pasó a poseer un sistema de disipación híbrido, puesto que el aerotermo inicial no fue eliminado de la instalación. La figura 1 muestra un esquema simplificado de la instalación.

\section{METODOLOGÍA}

El sistema de captación de datos se diseñó para poder llevar a cabo diferentes balances de energía y masa en cada uno de los componentes principales de la instalación. La metodología de captación de datos se fundamentó en la recogida de una serie de datos (temperatura de los flujos 
de agua, radiación solar, temperatura ambiente,..) (Monné et al, 2011). Una vez analizados y verificados estos datos, denominados variables primarias, se calcularon las variables secundarias necesarias para la realización del estudio del comportamiento energético de la máquina de absorción (potencias, rendimiento de los captadores solares, COP, etc.). Posteriormente se realizaron cuatro tipos diferentes de gráficos (de rango, de fase, estacionarios y de tendencia) para analizar del comportamiento de la instalación. De los cuatro tipos, tienen especial importancia los gráficos de tendencia.

Para la realización de las tendencias entre las diferentes variables se debe definir un estado estacionario de funcionamiento de la máquina. En este intervalo temporal se pueden comparar, manteniendo un mismo escenario de operación, el comportamiento que ha tenido la máquina en los días diferentes analizados. En los gráficos que aparecen en este documento se muestran las medias de los valores alcanzados en el período estacionario de las variables estudiadas.

\section{ANÁLISIS DE LA INSTALACIÓN}

Durante los años 2007 y 2008 se observó la influencia que presentaba la temperatura del foco de condensación (temperatura ambiente en este caso) en el la potencia térmica disipada por el aerotermo de la máquina. En la figura 2 se observa como existe una relación lineal entre el aumento de la $\mathrm{T}^{\mathrm{a}}$ ambiente y la disminución de la potencia disipada. La separación entre ambas líneas de tendencia se explica porque los caudales utilizados en la instalación fueron distintos en 2007 y 2008. Sin embargo, la pendiente característica de la instalación, es la misma en ambos casos.

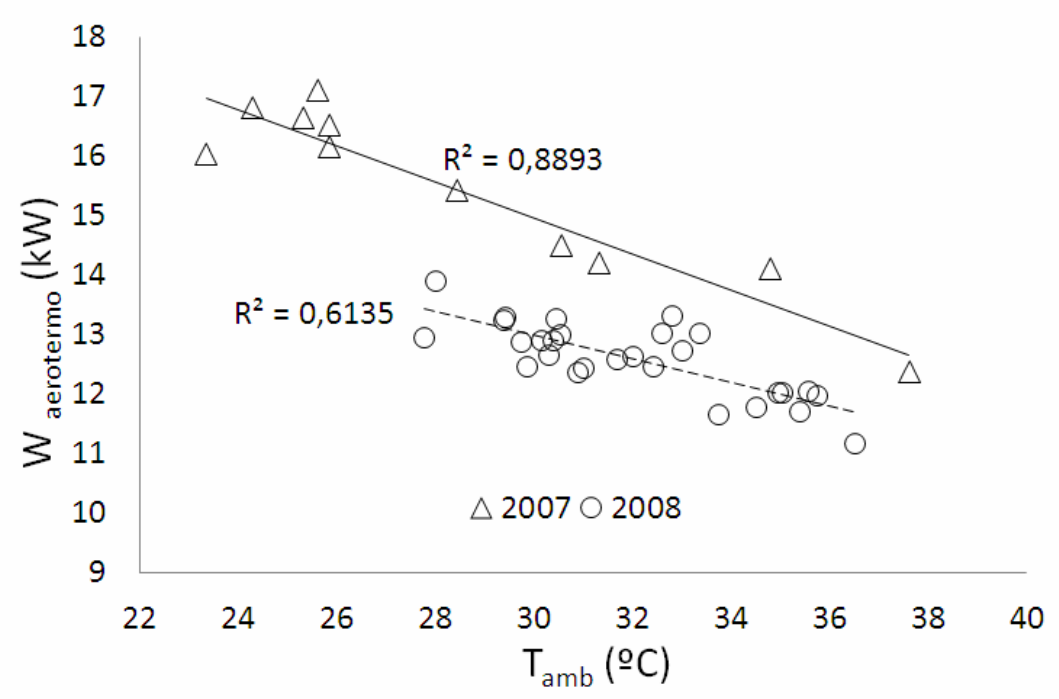

Fig. 2: Evolución de la potencia disipada en función de la temperatura ambiente.

Al aumentar la temperatura del foco frío, la cantidad de calor que se puede disipar al exterior disminuye, de este modo se empeoran las condiciones de transferencia de calor en el interior de la máquina de absorción. Esta situación conlleva un peor funcionamiento de la máquina y, consecuentemente, una disminución de la potencia de refrigeración producida. Este hecho queda patente en la figura 3 , donde se observa como a medida que aumenta la temperatura ambiente disminuye la producción de refrigeración del sistema. Nuevamente, existe una diferencia entre la tendencia de 2007 y 2008 debido a los caudales utilizados, pero la pendiente es similar para ambos grupos de datos. Los valores medios de las variables más importantes para estos dos años de funcionamiento de la instalación con el aerotermo se muestran en la tabla 1. Durante el año 2009, con el nuevo sistema de disipación geotérmica, se analizó nuevamente la instalación. 


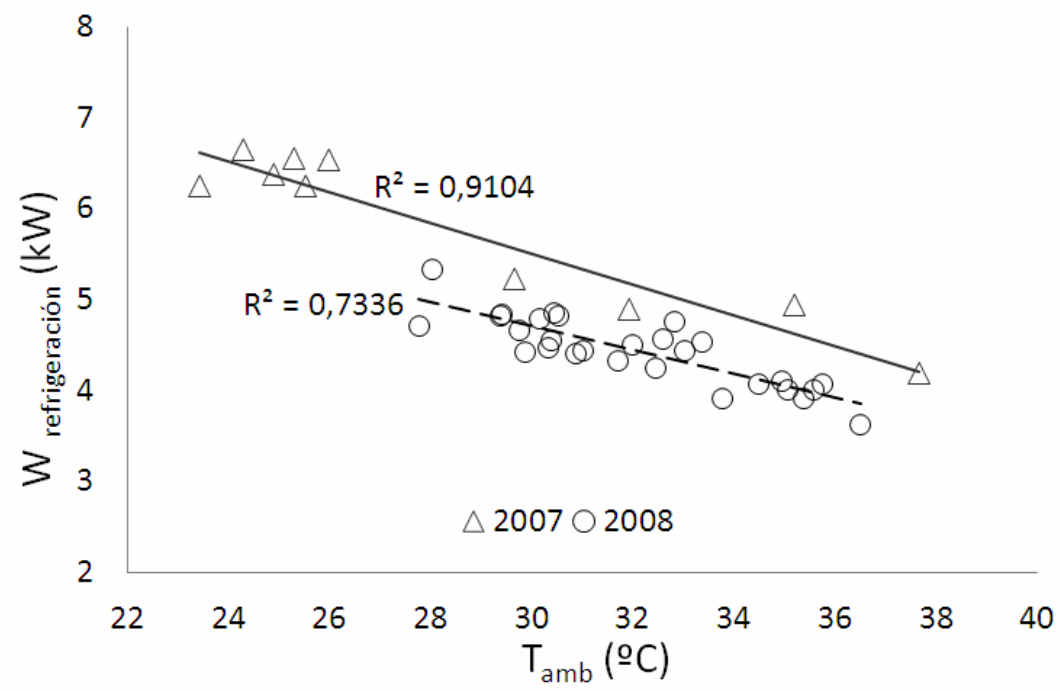

Fig. 3: Evolución de la potencia de refrigeración en función de la temperatura ambiente.

Tabla 1: Valores medios de potencia y COP en 2007 y 2008.

\begin{tabular}{ccccc}
\hline Año & $\mathrm{W}_{\text {refrigeración }}(\mathrm{kW})$ & $\mathrm{W}_{\text {generador }}(\mathrm{kW})$ & $\mathrm{W}_{\text {aerotermo }}(\mathrm{kW})$ & $\mathrm{COP}(-)$ \\
\hline 2007 & 5.8 & 9.7 & 15.4 & 0.56 \\
\hline 2008 & 4.4 & 8.0 & 12.5 & 0.51 \\
\hline
\end{tabular}

En la figura 4 se puede ver una comparación de la potencia de frío producida con uno y otro sistema. Hay tres series de datos representadas; con aerotermo, con caudal de $49 \mathrm{l} / \mathrm{min}$ en la disipación geotérmica o con caudal de $95 \mathrm{l} / \mathrm{min}$. Con el aerotermo se produce menos frío a medida que aumenta la temperatura ambiente, pero con el nuevo sistema de disipación esta producción es prácticamente constante. Esto es así porque antes se disipaba el calor a un foco de temperatura variable, pero ahora se tiene un foco de temperatura constante (el suelo y el tanque de agua) al que se le puede ceder siempre la misma cantidad de calor. Se observa que a mayor caudal en la disipación geotérmica, mayor es la potencia producida. Además, se ve como a partir de los $34^{\circ} \mathrm{C}$ de temperatura ambiente (punto de corte de dos líneas de tendencia), la producción de frío es mayor utilizando el sistema geotérmico que utilizando el aerotermo. En la tabla 2 se puede ver un resumen de los principales valores medios medidos en la instalación funcionando con el sistema de disipación geotérmico.

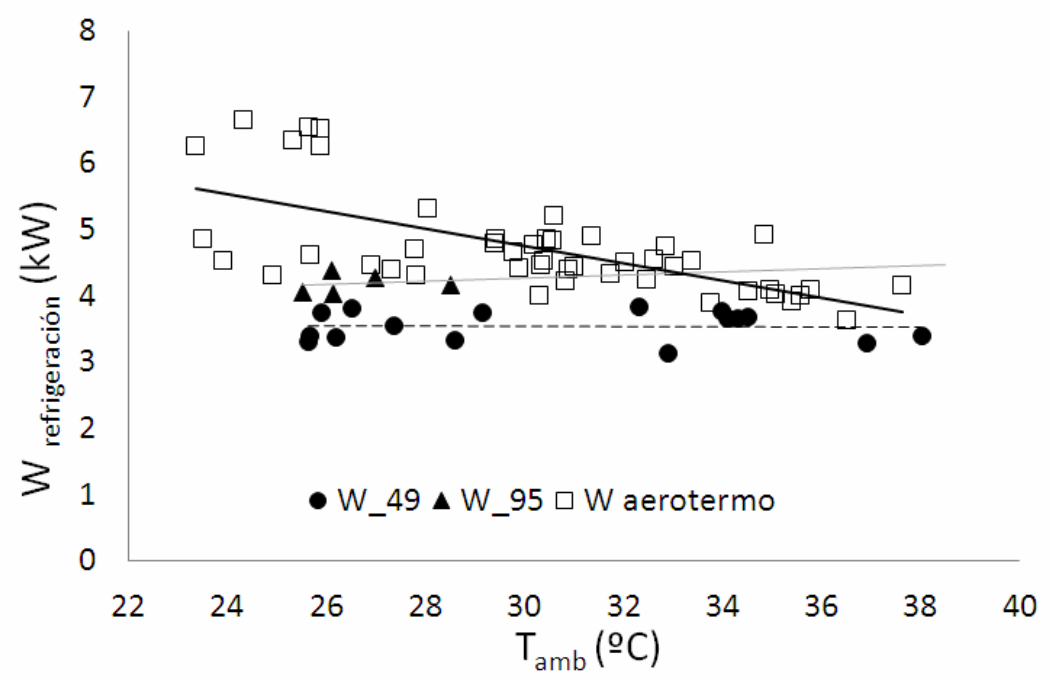

Fig. 4: Comparación de la potencia de refrigeración según el sistema de disipación utilizado. 
Tabla 2: Valores medios de potencia y COP con los dos caudales utilizado en el sistema geotérmico.

\begin{tabular}{rcccc}
\hline Caudal & $\mathrm{W}_{\text {refrigeración }}(\mathrm{kW})$ & $\mathrm{W}_{\text {generador }}(\mathrm{kW})$ & $\mathrm{W}_{\text {aerotermo }}(\mathrm{kW})$ & $\mathrm{COP}(-)$ \\
\hline $49 \mathrm{I} / \mathrm{min}$. & 3.5 & 7.1 & 10.5 & 0.47 \\
\hline $95 \mathrm{I} / \mathrm{min}$. & 4.2 & 7.6 & 12.3 & 0.52 \\
\hline
\end{tabular}

\section{CONCLUSIONES}

Tras el análisis de los datos se llega a la conclusión de que la instalación de refrigeración solar se comporta de un modo más uniforme utilizando un sistema de disipación geotérmico. Es decir, el uso de la geotermia como mecanismo de disipación de calor, consigue que el funcionamiento de la instalación sea independiente de las variaciones de temperatura ambiente, alcanzando en todo momento prestaciones constantes. Sin embargo, hay que destacar que para temperaturas ambiente bajas la disipación es más efectiva con el aerotermo. Por ello, se puede concluir que el uso de estos sistemas de disipación geotérmicos es más óptimo en aquellas zonas climáticas donde se den temperaturas medias más altas en épocas con demanda de refrigeración.

\section{REFERENCIAS}

Argiriou, A.A., Balaras, C.A., Kontoyiannidis, S., Michel, E. Numerical simulation and performance assesment of a low capacity solar assisted absorption heat pump coupled with a sub-floor system. Solar Energy 79, 290-301. (2005)

Assilzadeh, F., Kalogirou, S.A., Ali, Y., Sopian, K. Simulation and optimization of a LiBr solar absorption cooling system with evacuated tube collectors. Renewable Energy 30, 1143-1159. (2005)

Atmaca, I., Yigit, A. Simulation of solar-powered absorption cooling system. Renewable Energy 28, 1277-1293. (2003)

Balaras, C.A., y otros seis autores. Solar air conditioning in Europe-an overview. Renowable and Sustainable Energy Reviews 11, 299-314. (2007)

Balghouthi, M., Chahbani MH, Guizani A. Solar powered air conditioning as a solution to reduce environmental pollution in Tunisia. Desalination $185,105-110$. (2005)

García Casals, X. Solar absorption cooling in Spain: Perspectives and outcomes from the simulation of recent installations. Renewable Energy 31, 1371-1389. (2006)

Henning, H.M. Solar assisted air conditioning of buildings-an overview. Applied Thermal Engineering, 27, 1734-1749. (2007)

Herold, K.E., Radermacher, R., Klein S.A. Absorption chillers and heat pumps. Book 329 pages, CRS Press LLC, 2000 N.W. (1996)

Kaynakli, O., Kilic, M. Theoretical study on the effect of operating conditions on performance of absorption refrigeration system. Energy Conversion and Management 48, 599-607. (2007)

Monné, C., Guallar, J., Alonso, S., Palacín, F. Influencia de la velocidad de giro en las máquinas rotativas de absorción. XIV Congreso Ibérico y IX Congreso Iberoamericano de Energía Solar. Vigo, Galicia, España. (2008)

Monné, C., Alonso, A., Palacín, F., Serra, L. Monitoring and simulation of an existing solar powered absorption cooling system in Zaragoza (Spain). Applied Thermal Engineering, 31, 28-35. (2011)

Syed, A., y otros seis autores. A novel experimental investigation of a solar cooling system in Madrid. International Journal of Refrigeration 28, 859-871. (2005) 